\title{
The silencing complex SAS-I links histone acetylation to the assembly of repressed chromatin by CAF-I and Asf1 in Saccharomyces cerevisiae
}

\author{
Sebastiaan H. Meijsing and Ann E. Ehrenhofer-Murray ${ }^{1}$ \\ Otto-Warburg-Laboratorium, Max-Planck-Institut für Molekulare Genetik, D-14195 Berlin, Germany
}

\begin{abstract}
The acetylation state of histones plays a central role in determining gene expression in chromatin. The reestablishment of the acetylation state of nucleosomes after DNA replication and chromatin assembly requires both deacetylation and acetylation of specific lysine residues on newly incorporated histones. In this study, the MYST family acetyltransferase Sas2 was found to interact with Cac1, the largest subunit of Saccharomyces cerevisiae chromatin assembly factor-I (CAF-I), and with the nucleosome assembly factor

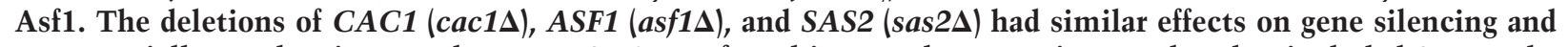
were partially overlapping. Furthermore, Sas2 was found in a nuclear protein complex that included Sas4 and Sas5, a homolog of $\mathrm{TAF}_{\text {II }} 3$. This complex, termed SAS-I, was also found to contribute to rDNA silencing. Furthermore, the observation that a mutation of $\mathrm{H} 4$ lysine 16 to arginine displayed the identical silencing phenotypes as sas2 $2 \Delta$ suggested that it was the in vivo target of Sas2 acetylation. In summary, our data present a novel model for the reestablishment of acetylation patterns after DNA replication, by which SAS-I is recruited to freshly replicated DNA by its association with chromatin assembly complexes to acetylate lysine 16 of $\mathrm{H4}$.
\end{abstract}

[Key Words: Silencing; histone acetylation; heterochromatin; acetyltransferase; PCNA]

Received July 25, 2001; revised version accepted October 4, 2001.

Replication of the eukaryotic genome involves the duplication not only of DNA but also of the chromatin in which the DNA is packaged in the nucleus. During this process, parental nucleosomes are distributed onto the daughter strands along with newly synthesized histones (Gruss et al. 1993). The replication of chromatin also requires the faithful inheritance of epigenetic chromatin marks to ensure appropriate gene expression in the daughter cells. One such mark is the acetylation state of lysine residues in the $\mathrm{N}$-terminal tails of the histones, which plays a central role in regulating the expression of genes in chromatin (Grunstein 1997). Although histone $\mathrm{H} 4$ is potentially acetylated at all four $\mathrm{N}$-terminal lysines, only a specific subset of these sites is modified in new histones when incorporated into freshly assembled chromatin (Smith and Stillman 1991). Thus, the reestablishment of parental acetylation states after chromatin replication requires both deacetylation and acetylation of specific lysine residues on newly incorporated histones. However, the mechanism by which assembly-

${ }^{1}$ Corresponding author.

E-MAIL ehrenhof@molgen.mpg.de; FAX 49-30-8413-1130.

Article and publication are at http://www.genesdev.org/cgi/doi/10.1101/ gad.929001. coupled changes in histone acetylation are achieved is currently not known.

Histone deposition after DNA replication is mediated by specialized histone chaperones. For example, the chromatin assembly factor-I (CAF-I) preferentially assembles nucleosomes onto replicating DNA (Stillman 1986). The association of CAF-I with the replication fork is mediated by its interaction with the proliferating cell nuclear antigen (PCNA; Shibahara and Stillman 1999). CAF-I is conserved in eukaryotic organisms ranging from yeast to man. CAF-I in Saccharomyces cerevisiae consists of three subunits, Cac1/Rlf2, Cac2, and Cac3/Msi1 (Kaufman et al. 1997). None of these are essential for cell viability, suggesting that other mechanisms of chromatin assembly exist. Interestingly, cac $\Delta$ mutants have defects in the stable inheritance of gene silencing at telomeres and at the silent mating-type loci, (Enomoto et al. 1997; Enomoto and Berman 1998), and several studies argue that the role of CAF-I in epigenetic control of gene expression is conserved (Murzina et al. 1999; Tchenio et al. 2001). Furthermore, cacs mutants are sensitive to ultraviolet irradiation, which likely is a reflection of the role of CAF-I in DNA repair (Kaufman et al. 1997). The function of CAF-I partially overlaps with that of the nucleosome assembly factor Asf1 in yeast. The Drosophila homolog of Asf1 was purified as a factor that 
enhances the nucleosome assembly activity of CAF-I in vitro (Tyler et al. 1999). Deletions of ASF1 and CAC1 synergistically decrease telomeric and $H M R$ silencing and increase sensitivity to mutagens (Tyler et al. 1999). Interestingly, specific mutations in yeast PCNA (e.g., pol30-8) abrogate the effect of cac1s on telomeric silencing, whereas other mutations (pol30-6 and pol30-79) abolish the effect of Asf1, suggesting that PCNA is central to the effect of both CAF-I and Asf1 in gene repression (Sharp et al. 2001). Several other observations indicate a link between DNA replication and silencing in yeast, including a role for the PCNA-loading protein, replication factor-C, $\mathrm{Cdc} 45$, and polymerase $\varepsilon$ in silencing (Ehrenhofer-Murray et al. 1999). The involvement of replication proteins in silencing may be related to the inheritance of silenced chromatin structures.

Transcriptional repression in yeast is found at the silent mating-type loci $H M L$ and $H M R$, at the telomeres and the ribosomal DNA locus (rDNA) (Sherman and Pillus 1997). Several connections are found between gene silencing and histone acetylation at these loci. In the repressed state, the majority of histones at the $H M$ loci are in their unacetylated form (Braunstein et al. 1996), which is required for the silencing factors Sir3 and Sir4 to interact with the histone tails and to bind chromatin at the HM loci (Hecht et al. 1995). Also, the $\mathrm{NAD}^{+}$-dependent histone deacetylase Sir2 is essential for all forms of yeast silencing (Moazed 2001). Furthermore, mutations in the structural gene for the histone acetyltransferase homolog Sas2 were isolated in a genetic screen for suppressors of silencing defects at HMR (EhrenhoferMurray et al. 1997). Sas2 belongs to the MYST family of histone acetyltransferases. Homologs include the yeast proteins Sas3 and Esa1, Drosophila MOF, and the human homologs Tip60, HBO1, and MOZ, which contributes to malignant transformation in acute myeloid leukemia (Sterner and Berger 2000). Interestingly, Sas2 has the opposite effects at $H M L$ and telomeric silencing compared with its effect at $H M R$, because the deletion of SAS2 (sas $2 \Delta$ ) decreases $H M L$ silencing in sir1s strains and causes telomeric derepression (Reifsnyder et al. 1996; Ehrenhofer-Murray et al. 1997). Two other genes have been identified to share their effect on silencing with SAS2: SAS4 encodes a cullin family protein, and SAS5 encodes a homolog of $\mathrm{TAF}_{\mathrm{II}} 30$ (Xu et al. 1999).

In this study, we investigated the cellular role of Sas2 and found it in a nuclear complex termed SAS-I that included Sas4 and Sas5. Importantly, this complex was found to interact with Cac1, but not the whole CAF-I complex, and with Asf1. Genetic studies suggested overlapping functions of Cacl and Asf1 that mediated the effect of Sas2 on silencing. Furthermore, a mutation in Lys 16 of histone $\mathrm{H} 4$ phenocopied the silencing effect of sas $2 \Delta$, suggesting that this lysine is the target of Sas2 acetylation in vivo. In summary, our data propose a novel mechanism for the reestablishment of histone acetylation patterns after DNA replication, in which SAS-I is recruited to the replication fork by its association with Cac1 and Asf1 to acetylate Lys 16 of histone $\mathrm{H} 4$ in chromatin.

\section{Results}

Identification of Sas2, Sas4, and Sas5 as components of the SAS-I complex

The MYST family acetyltransferase homolog Sas2 was originally identified in a genetic screen for factors that, when mutated, restored repression to a silencing-defective HMR locus (Ehrenhofer-Murray et al. 1997). To identify proteins that interact with Sas2, we performed a two-hybrid screen (Chien et al. 1991). One interacting clone specific for Sas 2 contained a fragment of the SAS4 gene (amino acids 4-329; Table 1). Interestingly, the deletion of SAS4 (sas4D) and SAS5 (sas5 5 ) has been shown previously to result in the identical silencing phenotypes as sas $2 \Delta$ (Xu et al. 1999), suggesting that the three proteins might be present in a protein complex. Therefore, we tested two-hybrid interactions of Sas5 with Sas2 and Sas4 and found that it interacted with Sas4, but not Sas2 (Table 1).

To test the relevance of these interactions in vivo, coimmunoprecipitation experiments were performed with fully functional epitope-tagged versions of Sas 4 and Sas5 with Sas2. In these experiments, any one of the three proteins coimmunoprecipitated with both other proteins (Fig. 1A). Notably, Sas2 and Sas5 coimmunoprecipitated, although they did not interact in the two-hybrid assay, suggesting that their interaction in the coimmunoprecipitation was bridged by Sas4. To test the possibility that the coimmunoprecipitation was DNA mediated, immunoprecipitations were conducted in the presence of high concentrations of ethidium bromide. Sas 2 and Sas4 still coimmunoprecipitated under this condition (data not shown), showing that their interaction was not DNA dependent.

To determine the apparent molecular mass of Sas2-, Sas4-, and Sas5-containing complexes in yeast, whole cell lysates of strains expressing myc-tagged versions of Sas2 and Sas4 and HA-tagged Sas5 were applied directly to a Sephacryl S-300 gel-filtration column. Column frac-

Table 1. Two-hybrid interactions between SAS-I components and chromatin assembly factors

\begin{tabular}{lcccc}
\hline & $\begin{array}{c}\text { Sas2- } \\
\text { GAD }\end{array}$ & $\begin{array}{c}\text { Sas4- } \\
\text { GAD }\end{array}$ & $\begin{array}{c}\text { Cac1- } \\
\text { GAD }\end{array}$ & $\begin{array}{c}\text { Asf1- } \\
\text { GAD }\end{array}$ \\
\hline LexA-Sas2 & + & + & + & + \\
LexA-Sas4 & + & + & + & $+^{\mathrm{a}}$ \\
LexA-Sas5 & - & + & + & + \\
LexA-Sas2 $\left(\mathrm{HAT}^{-}\right)$ & n.d. & - & - & n.d. \\
LexA-Sas2 $\left(\mathrm{Zn}^{-}\right)$ & n.d. & - & - & n.d. \\
LexA & - & - & - & - \\
\hline
\end{tabular}

The reporter strain (L40c) was transformed with a DNA-binding domain plasmid (LexA fusions) and a Ga14 activation domain plasmid (GAD). (For plasmids, see Table 3.) Interactions were measured as activation of the HIS3 and lacZ reporter genes. $(+)$ Transformants that became blue in a filter assay with X-Gal within 15 to $30 \mathrm{~min}$ and were able to grow on plates lacking histidine; (-) colonies that were not blue after $>2 \mathrm{~h}$ and did not grow on plates lacking histidine; (n.d.) not determined.

a Sutton et al. 2001. 

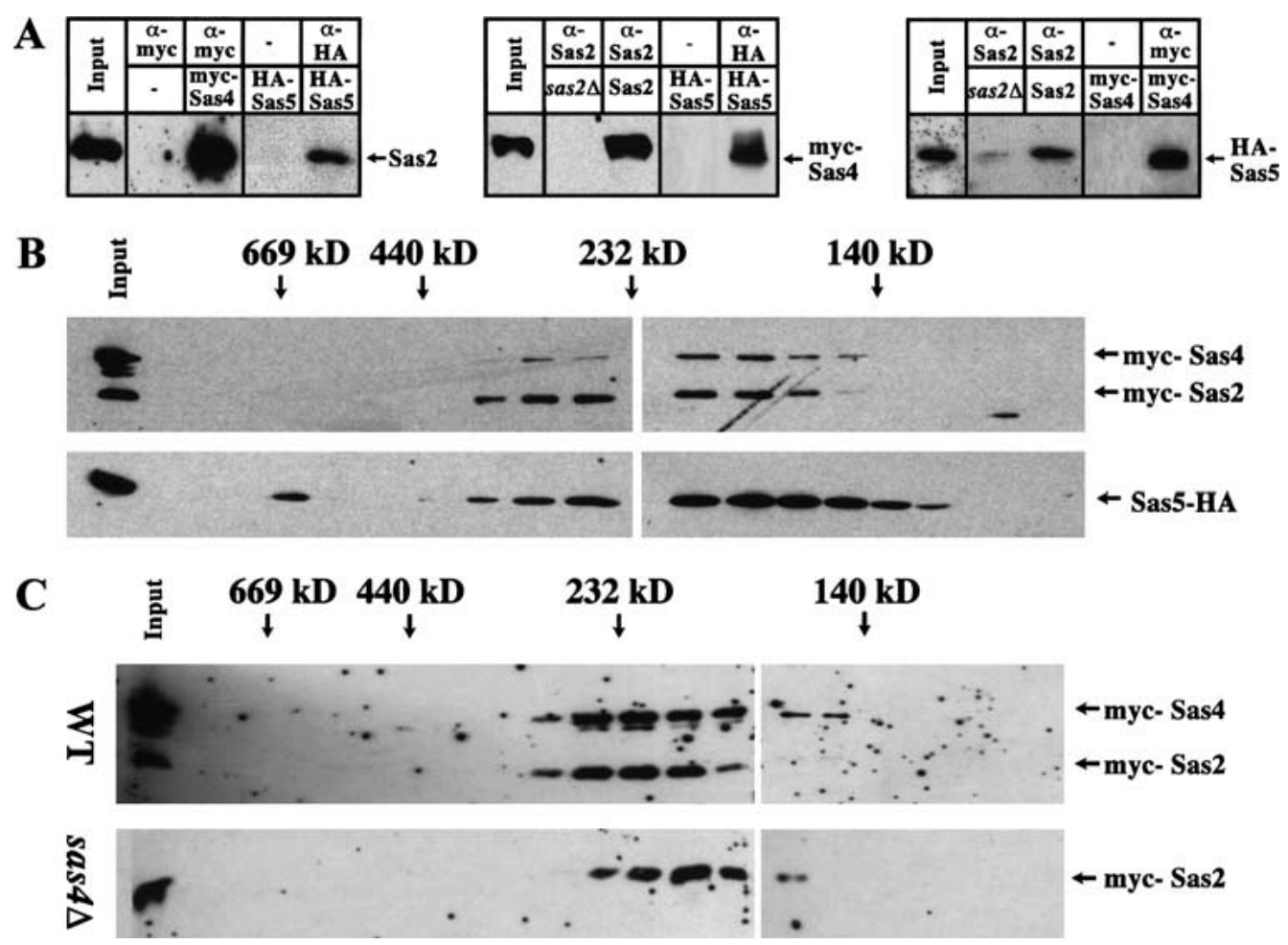

Figure 1. (A) Interactions between Sas2, Sas4, and Sas5. (Left) Sas2 coimmunoprecipitated with myc-Sas4 and HA-Sas5. Coimmunoprecipitations were with strain AEY1558 transformed with pAE240 (Sas2) and with pAE612 (myc-Sas4) or pAE625 (HA-Sas5). Immunoprecipitations were probed for Sas2 with an anti-Sas2 antibody. (Center) myc-Sas 4 coimmunoprecipitated with Sas 2 and HA coimmunoprecipitated Sas5. For coimmunoprecipitation of myc-Sas4 with Sas2, extracts from strain AEY1559 transformed with pRS315 (sas24) or pAE240 (Sas2) and pAE612 (myc-Sas4) were immunoprecipitated with anti-Sas2 antibody ( $\alpha$-Sas2). For coimmunoprecipitation of myc-Sas4 with HA-Sas5, AEY1559 transformed with pAE613 (myc-Sas4) and pAE625 (HA-Sas5) was used. Precipitates were analyzed by immunoblotting with an $\alpha$-myc antibody. (Right) Sas5 coimmunoprecipitated with Sas 2 and myc-Sas4. Immunoprecipitation between Sas2 and HA-Sas 5 was performed in extracts from strain AEY1559 transformed with pRS315 (sas24) or pAE240 (Sas2) and pAE625 (HA-Sas5). Immunoprecipitation between myc-Sas4 and HA-Sas5 was performed in strain AEY1558 transformed with pAE613 and pAE625. Immunoblotting of immunoprecipitations with $\alpha$-HA was used to detect HA-Sas5. (B) Sas2, Sas4, and Sas5 coeluted from a gel filtration column. Elution profile of low-copy myc-Sas2, myc-Sas4, and Sas5-HA on a Sephacryl S300 column. The strain used was AEY2465 transformed with pAE778 (myc-Sas2) and pAE779 (myc-Sas4). Fractions were analyzed by immunoblotting with an $\alpha$-myc (top) and an $\alpha$-HA antibody (bottom). The elution peaks of marker proteins are indicated above. (C) Deletion of Sas4 altered the elution profile of the SAS-I complex. Strain AEY2424, transformed with pAE778 (myc-Sas2) and pAE779 (myc-Sas4; WT, top) or with pAE778 (myc-Sas2) only (sas44, bottom) was used for gel-filtration experiments.

tions were analyzed by SDS-PAGE and immunoblotting (Fig. 1B). This analysis indicated that Sas2, Sas4, and Sas 5 coeluted in a peak of $220 \mathrm{kD}$. A minor part of Sas5, but not Sas2 or Sas4, was also found in higher molecular mass fractions, arguing that Sas 5 might be part of one or more protein complexes. Furthermore, the elution profile of Sas2 was shifted toward a peak of lower molecular mass $(\sim 190 \mathrm{kD})$ in extracts from a sas $4 \Delta$ strain (Fig. 1C), suggesting that Sas4 was part of Sas2-containing complex(es) but that macromolecular complexes of Sas 2 existed in the absence of Sas4. The calculated molecular masses of myc-Sas2, myc-Sas4, and Sas5 add up to $\sim 140$ $\mathrm{kD}$, indicating that the Sas2/Sas4/Sas5 complex may contain additional subunits. Alternatively, there may be multimers of single components present in these complexes. Notably, Sas2 interacts with itself in two-hybrid assays, suggesting that the complex may contain multiple Sas2 subunits. The complex containing Sas2, Sas4, and Sas5 was termed SAS-I.
Sas2 was a chromatin-bound nuclear protein involved in $r D N A$ silencing

S. cerevisiae has three types of silenced loci, the HM loci, the telomeres, and the rDNA locus. The effects of Sas2, Sas4, and Sas 5 have been described at the HM loci and the telomeres. To determine their effect on rDNA silencing, we tested the effect of the respective deletions on repression of an $U R A 3$ gene integrated at the rDNA locus (Smith and Boeke 1997). The deletion of SAS2, SAS4, or SAS5 resulted in stronger URA3 repression (Fig. 2A), showing that the deletions increased rDNA repression. sas $2 \Delta$ also increased repression of MET15 when integrated in the rDNA locus (Fig. 2B; Smith and Boeke 1997), showing that the effect of sas $2 \Delta$ was independent of the rDNA-silencing reporter gene. Furthermore, the increased repression of MET15 by sas $2 \Delta$ depended on the presence of SIR2 and thus likely reflected a silencing effect of sas $2 \Delta$ rather than, for example, a loss of MET15 
A

B WT

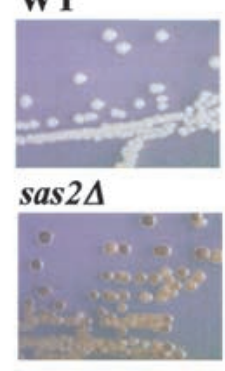

$\operatorname{sir} 2 \Delta$

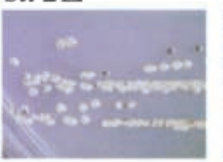

$\operatorname{sir} 2 \Delta \operatorname{sas} 2 \Delta$

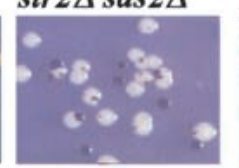

$\operatorname{cac1\Delta }$

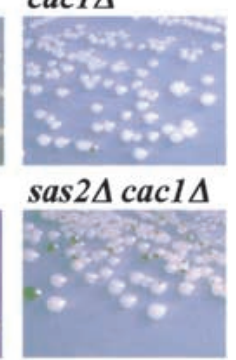

C

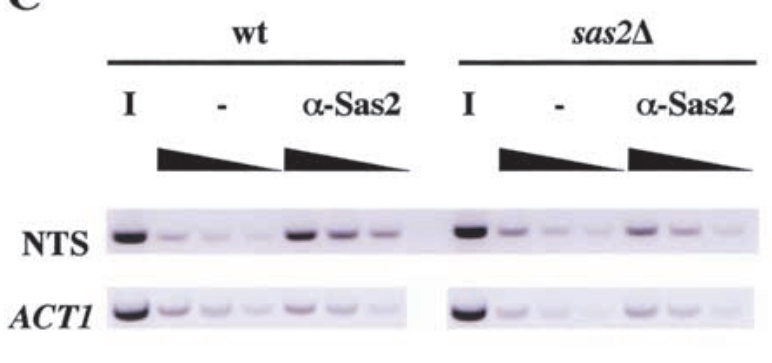

5-FOA
D

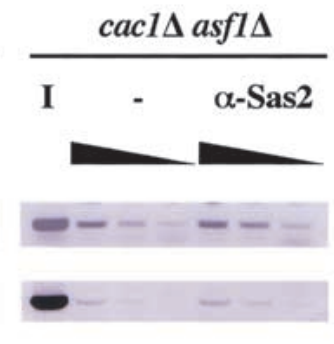

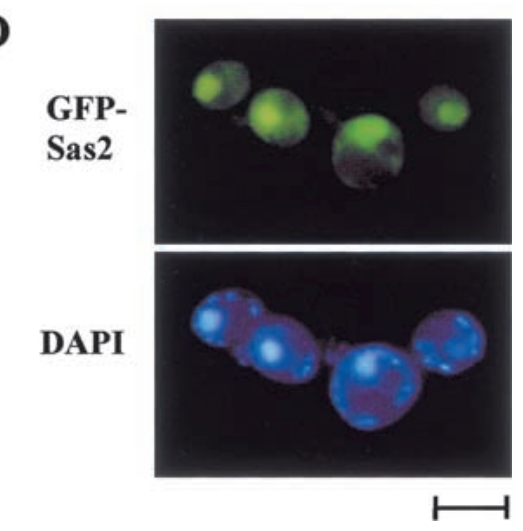

Figure 2. SAS-I was involved in rDNA silencing. (A) The deletion of SAS2, SAS4, or SAS5 increased repression of URA3 inserted in the rDNA locus. Silencing of URA3 was measured as growth on 5-FOA-containing medium. The strains used were AEY1778, AEY2279, AEY2360, and AEY2361 (from top to bottom). (B) Increased silencing of MET15 by sas2D in rDNA required SIR2. Strains were assayed for MET15 activity by streaking them on lead indicator medium. The strains used were AEY1201 (WT), AEY1202 (sir2A),

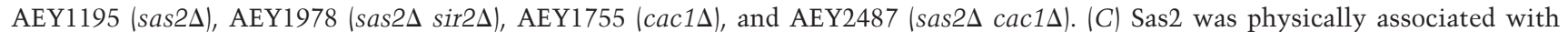
rDNA sequences. Chromatin immunoprecipitations (CHIPs) were performed with extracts from a wild type (AEY1), a sas2 $\Delta$ strain (AEY269), and a cac1s asf11s (AEY2451) strain using $\alpha$-Sas2 or no antibody (-). PCR was performed with increasing amounts of precipitate (black triangle) using primers specific to the nontranscribed spacer of RDN1 (NTS) and ACT1. The inverse image of PCR products resolved on ethidium bromide-stained $1 \%$ agarose gels is shown. (D) GFP-tagged Sas2 localized to the nucleus. The sas $2 \Delta$ strain AEY474 was transformed with pAE94. Bar, $5 \mu \mathrm{m}$.

activation, because MET15 expression and rDNA recombination increased in a sas $2 \Delta \operatorname{sir} 2 \Delta$ strain (Fig. 2 B).

We next tested whether the effect of Sas 2 on rDNA silencing was direct by determining whether Sas 2 was physically present at rDNA. Chromatin immunoprecipitations (CHIPs) were performed with wild-type or sas $2 \Delta$ strains. In these experiments, rDNA sequences from the nontranscribed spacer region of the rDNA locus were weakly but reproducibly enriched in the precipitates from the wild-type strain but not from the sas $2 \Delta$ strain, whereas ACT1 sequences were not specifically amplified (Fig. 2C). These results showed that Sas2 was present at rDNA sequences, thus arguing for a direct role of Sas 2 in gene expression at the rDNA locus. The relatively mild enrichment may reflect cell cycle-dependent binding of Sas2 to rDNA sequences.

We further determined the subcellular localization of Sas2 in living cells by determining the localization of Sas2 fusions to green fluorescent protein (GFP) within the cell. GFP signals were only detectable on overexpression of the fusion protein. A fully functional fusion of GFP to the C terminus of Sas2 predominantly stained the nucleus, including the nucleolus (Fig. 2D), thus showing a nuclear localization for Sas2.
Sas2 function required the acetyl-CoA binding site and the atypical zinc finger

As a member of the MYST family of acetyltransferases, Sas2 contains two recognizable motifs (Fig. 3A): (1) an acetyl-CoA binding site (HAT) that is homologous to acetyltransferases within and outside of the MYST family (e.g., Gen5 and Hat1), and (2) an atypical zinc finger (CCHC, $\mathrm{Zn}$ ), which is found in the MYST family proteins and is necessary for substrate recognition in Drosophila MOF (Akhtar and Becker 2001). We tested the functional relevance of these motifs in Sas 2 by introducing point mutations in these domains and determining their effect on the function of Sas2. The deletion of SAS2 results in a loss of $H M L$ silencing in $\operatorname{sir} 1 \Delta$ strains and in derepression of telomeric URA3. At $H M R$, sas2 $2 \Delta$ improves repression at $H M R$ alleles carrying mutations in the HMR-E silencer $\left(H M R \mathbf{a}-\mathrm{e}^{\star \star}\right.$; Ehrenhofer-Murray et al. 1997). Both a mutation in the putative zinc finger $\left(\mathrm{C} 160 \mathrm{~L}, \mathrm{Zn}^{-}\right)$or in the acetyl-CoA binding site of Sas2 (P213A/P214V, $\left.\mathrm{HAT}^{-}\right)$resulted in a complete loss of complementation (Fig. 3B,C). This was not caused by a loss of expression or stability of the mutant Sas 2 proteins, because equal amounts of the mu- 
A MYST homology domain

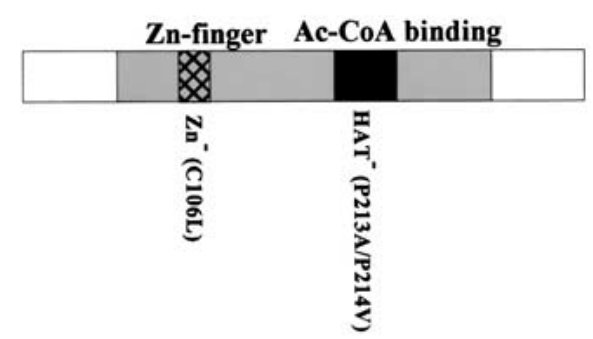

C

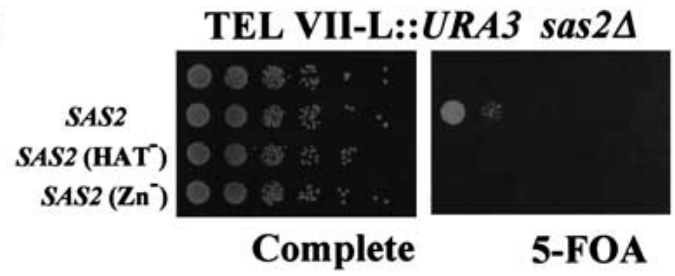

B MATa $\operatorname{sir} 1 \Delta \operatorname{sas} 2 \Delta \quad M A T \alpha H M R a-e^{* *} \operatorname{sas} 2 \Delta$

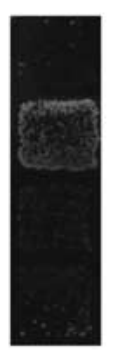

D

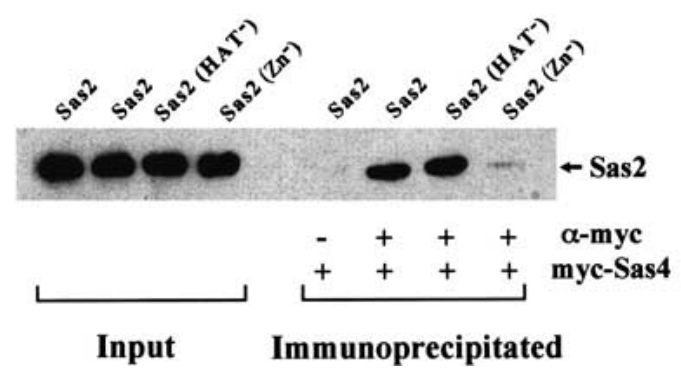

Figure 3. Mutations in the HAT domain and the putative zinc finger disrupted functions of Sas2 in silencing. (A) Schematic representation of Sas2. (Gray) Region of Sas2 homologous to other MYST family proteins; (cross-hatched) the CCHC zinc finger; (black) acetyl-CoA-binding site (HAT). The mutations in the zinc finger $\left(\mathrm{Zn}^{-}\right)$and the acetyl-CoA-binding domain $\left(\mathrm{HAT}^{-}\right)$are indicated. $(B)$ Point mutations in the HAT and zinc finger domains disrupt the function of Sas2 in HM silencing. Silencing of AEY474, a MAT $\alpha$

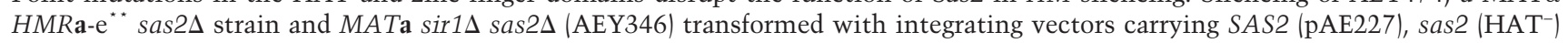
(pAE230), sas2 ( $\mathrm{Zn}^{-}$) (pAE389), or no insert (pRS303) was monitored by patch-mating assays using MATa his4 or MATa his4 as mating tester lawns, respectively. $(C)$ Mutations in Sas2 disrupted its ability to function in telomeric silencing. Silencing of a URA3 gene inserted near the left telomere of chromosome VII was tested in serial dilution assays on 5-FOA-containing medium. The strains used were derivatives of AEY1190 transformed with plasmids as in $B .(D)$ The CCHC zinc finger of Sas2 was essential for its interaction with Sas4. Cell extracts were prepared from AEY2424 expressing myc-Sas4 and wild type (pAE240) or mutant Sas2 (HAT ${ }^{-}$, pAE321 and $\left.\mathrm{Zn}^{-}, \mathrm{pAE} 491\right)$. Sas4 was precipitated with $\alpha$-myc, and the precipitates were immunoblotted using $\alpha$-Sas 2 .

tant Sas2 proteins were detected in immunoblots (Fig. 3D).

We further tested whether these mutations affected the interaction of Sas2 with Sas4. We found that mutations in either domain abolished the two-hybrid interaction between Sas2 and Sas4 (Table 1). Interestingly, the zinc finger mutation, but not the acetyl-CoA binding site mutation, resulted in a loss of the ability of Sas 4 to coimmunoprecipitate Sas2 (Fig. 3D). Perhaps the three-dimensional folding of the proteins in the two-hybrid fusions is disturbed such that an additional HAT mutation has a larger effect on the fusion than on the native protein. Taken together, these results suggested that the zinc finger of Sas2 was essential for the interaction with Sas4, whereas a mutation in the acetyl-CoA binding site likely abrogated the enzymatic activity of Sas2. Notably, zinc finger mutations in MOF and Sas3 have been shown to result in a loss of enzymatic activity (Takechi and Nakayama 1999; Akhtar and Becker 2000).

\section{A mutation in histone H4 (H4K16R) phenocopied the effect of sas $2 \Delta$ in silencing}

Sas2 is highly homologous to Drosophila MOF, which preferentially acetylates Lys 16 of histone H4 (Akhtar and Becker 2000). However, we were unable to detect in vitro activity for Sas2 (data not shown), although the homology comparison and mutation analysis (see above) argue that Sas 2 is a (histone) acetyltransferase. We therefore performed a set of genetic experiments to determine which lysine residue(s) in the $\mathrm{N}$-terminal tail of $\mathrm{H} 4$ might be the target for Sas2 in vivo. Acetylation at the $\varepsilon-\mathrm{N}$ position of lysine side chains neutralizes their positive charge. Thus, if Sas2 is specific for a particular lysine residue, the mutation of this residue to a positively charged arginine is expected to imitate the effect of the deletion of SAS2. We constructed strains carrying as their sole copy of the H4 gene HHF1 an allele with mutations that changed lysines 5 and $8(\mathrm{~K} 5,8 \mathrm{R}), 5$ and 12 $(\mathrm{K} 5,12 \mathrm{R})$, or lysine 16 to arginine (K16R) and tested whether these alleles phenocopied the effect of sas $2 \Delta$ on silencing at $H M R, H M L$, and the telomeres.

Significantly, only H4 K16R was capable of imitating the effect of sas $2 \Delta$. We found that H4 K16R suppressed silencing defects at a mutated $H M R$ allele (Fig. 4A). The most direct explanation of this result is that Sas2 acetylates $\mathrm{H} 4 \mathrm{~K} 16$, which is detrimental to silencing at a mutated $H M R$ allele. Thus, in the absence of the acetyltransferase, or when this position in the $\mathrm{H} 4$ tail is occupied by a positively charged amino acid, silencing is improved. Furthermore, H4 K16R caused derepression at $H M L$ in a $\operatorname{sir} 1 \Delta$ strain (Fig. 4B) and telomeric URA3 (Fig. 


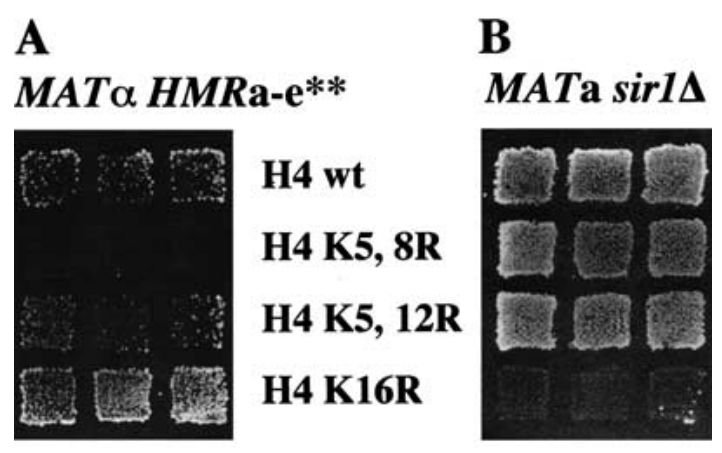

C

TEL VII-L:: URA3

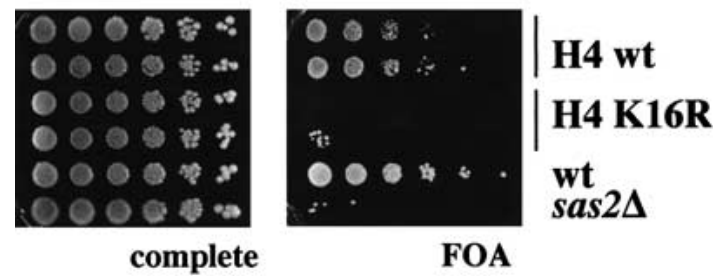

D MAT $\alpha$ HMRa-e**

H4 wt

H4 K16R

H4 K16R sas2 $\Delta$

Figure 4. A mutation of lysine 16 to arginine in histone $\mathrm{H} 4$ displayed the same silencing phenotypes as sas $2 \Delta$ at $H M R$, $H M L$, and the telomeres. (A) H4 K16R restored silencing at a defective $H M R$ allele. The panel shows the $\alpha$-mating ability of MAT $\alpha H M R \mathbf{a}-\mathrm{e}^{* *}$ strains carrying various $\mathrm{H} 4$ alleles. The strains used were AEY 1976 (wt), AEY1974 (K5, 8R), AEY2197 (K5, 12R), and AEY1956 (K16R). (B) H4 K16R caused HML derepression in a sir $1 \Delta$ strain. The a-mating ability of the MATa sir1 strains AEY2221 (WT), AEY2222 (K5, 8R), AEY2223 (K5, 12R), and AEY2224 (K16R) is shown. (C) H4 K16R caused derepression of telomeric URA3. Silencing of URA3 was measured in serial dilution assays on 5-FOA medium. The strains used were AEY2302 (H4 wt), AEY2304 (H4 K16R), AEY1017 (wt), and AEY1190 (sas2D). (D) Additional deletion of SAS2 did not alter the suppression of $H M R \mathbf{a}-\mathrm{e}^{* *}$ by H4 K16R. The $\alpha$-mating ability

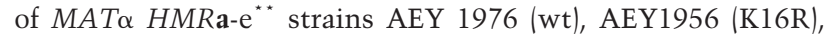
and AEY2475 (K16R sas24) is shown.

4C), as does the deletion of SAS2. Notably, the $H M L$ derepression completely depended on $\operatorname{sir} 1 \Delta$, because no derepression was observed in SIR1 strains (data not shown).

If the H4 K16R mutation and sas $2 \Delta$ have identical phenotypes because H4 K16 is the target of Sas 2 in vivo, a sas2 $2 \mathrm{H} 4 \mathrm{~K} 16 \mathrm{R}$ double mutant is expected not to be more mating proficient than the H4 K16R mutation alone. Therefore, we tested the effect of sas $2 \Delta$ on silencing of the mutated $H M R$ locus in the H4 K16R strain. Silencing was not further affected in this strain, arguing that the effect of $S A S 2$ on silencing was mediated by Lys
16 H4 (Fig. 4D). Similarly, telomeric silencing was equally derepressed in H4K16R SAS2 and sas2D strains (data not shown).

The SAS-I complex interacted with Cac1, the largest subunit of CAF-I

In a two-hybrid screen with Sas2, we also identified Cac1 as an interacting partner (amino acids $80-517$ ). Cacl is the largest subunit of the widely conserved CAF-I that performs replication-coupled chromatin assembly and consists of two more subunits, Cac2 and Cac3 (Kaufman et al. 1997). Cac1 also interacted with Sas4 and Sas5 in two-hybrid assays, suggesting that it interacted with the whole SAS-I complex (Table 1). In coimmunoprecipitation experiments, Cacl immunoprecipitated with both Sas2 and Sas4 (Fig. 5A). This interaction was not mediated by DNA, because the proteins still interacted in the presence of ethidium bromide (data not shown). However, Cacl was not an integral part of the SAS-I complex, because the elution profiles of myc-tagged Sas 2 and Sas 4 from a $\operatorname{cac} 1 \Delta$ strain on a gel filtration column were identical to those of a wild-type strain (data not shown).

To determine whether SAS-I interacted with the whole CAF-I complex or with the Cacl subunit only, we

A
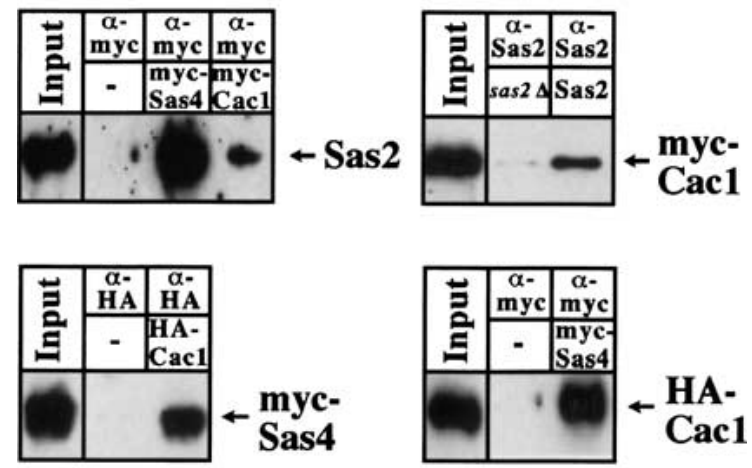

B

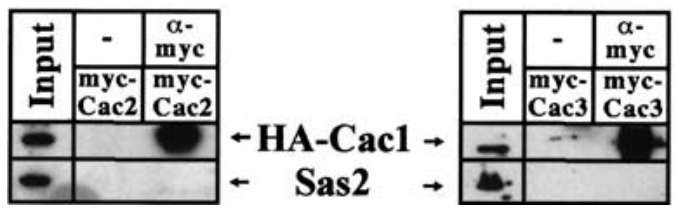

Figure 5. Sas2 and Sas 4 interacted with Cac1, but not Cac2 or Cac3. (A) Sas2 and Sas4 coimmunoprecipitated with Cac1. Cell extracts were prepared from AEY1558 or AEY1559 expressing myc-Cac1 (pAE614) or HA-Cac1 (pAE544) and myc-Sas4 (pAE613) or Sas2 (pAE240), as appropriate; precipitated with antibodies as indicated; and analyzed by immunoblotting. $(B)$ $\mathrm{Cac} 2$ and $\mathrm{Cac} 3$ coimmunoprecipitated Cac1, but not Sas2. Extracts from strain AEY1558 expressing HA-Cac1 (pAE544), myc-Cac2 (pAE820), and Sas2 (pAE240) or strain AEY2463 (Cac3-myc) expressing HA-Cac1 (pAE544) and Sas2 (pAE240) were immunoprecipitated with $\alpha$-myc and immunoblotted with $\alpha$-HA (top) or $\alpha$-Sas2 (bottom). 
attempted to coimmunoprecipitate other subunits of CAF-I, Cac2, or Cac3 with Sas2, Sas4, or Sas5. For this purpose, Cac2 or Cac3 were epitope tagged, and the precipitates were tested for the presence of CAF-I and SAS-I proteins. Significantly, in immunoprecipitation with Cac2 or Cac3, Cac1 was precipitated, but not Sas2 (Fig. 5B), Sas4, or Sas5 (data not shown). Similarly, Cac2 was not precipitated in immunoprecipitations with SAS-I proteins (data not shown). This suggested that only Cac1 rather than the CAF-I complex interacted with SAS-I.

The observation that the SAS-I complex interacted with Cac1 suggested that SAS-I is recruited to particular sites of the genome by Cacl and acetylates histones on freshly assembled chromatin. To test this, we conducted CHIPs with Sas2 in a cac1s strain that was also deleted for the nucleosome assembly factor ASF1 (see below). Sas2-dependent enrichment of rDNA sequences was lost in this strain (Fig. 2C), arguing that the recruitment of Sas2 to this locus was mediated by its interaction with chromatin assembly factors.

CAF-1 has roles both in replication-coupled chromatin assembly and in DNA repair, which is reflected in the increased sensitivity of cac mutants to ultraviolet irradiation (Kaufman et al. 1997). However, sas2s strains were no more ultraviolet sensitive than were isogenic wild-type strains (data not shown), suggesting that the role of SAS-I was related to the role of Cac1 in replication-coupled chromatin assembly rather than in DNA repair.

The effects of cac1 $1 \Delta$ and sas $2 \Delta$ on silencing were similar and partially overlapping

The deletion of CAF-I subunits affects gene silencing in S. cerevisiae (Kaufman et al. 1997; Enomoto and Berman 1998). If one of the functions of Cacl was to recruit the SAS-I complex to duplicated chromatin, one prediction would be that the deletion of CAC1 has similar phenotypes as the deletion of SAS2, SAS4, or SAS5 and that $\operatorname{cac} 1 \Delta$ and sas $2 \Delta$ are epistatic to each other. Cac1 has previously been shown to be redundant with the histone regulator protein Hirl and with the silencing establishment factor Sirl for silencing at HML (Enomoto and Berman 1998; Kaufman et al. 1998). Strong derepression by cac1s is only seen in strains mutated in both HIR 1 and SIR1. This phenotype is reminiscent of the effect of $\operatorname{sas} 2 \Delta$ at $H M L$, which only causes derepression in $\operatorname{sir} 1 \Delta$ strains. Also, both cac1s and sas $2 \Delta$ cause telomeric derepression. To test epistasis of cac $1 \Delta$ and sas $2 \Delta$, silencing at $H M L$ was measured in $\operatorname{sir} 1 \Delta$ strains carrying both cac1 $\Delta$ and sas $2 \Delta$. The additional deletion of CAC1 had no effect on $H M L$ silencing in a sir1 $1 \Delta$ sas $2 \Delta$ strain (Fig. 6A). In quantitative mating assays, the mating efficiency of a MATa sir1D sas $2 \Delta$ strain was $1.9 \times 10^{-4} \pm 0.7 \times 10^{-4}$ compared with that of a wild-type MATa strain, and the mating efficiency of a strain with an additional CAC1 deletion was $2.1 \times 10^{-4} \pm 1.3 \times 10^{-4}$. The roles of $C A C 2$ and $C A C 3$ at the $H M L$ locus were also epistatic with $S A S 2$, because neither cac $2 \Delta$ nor cac3s leads to a further decrease of silencing in a MATa sir1 1 sas $2 \Delta$ strain (Fig. 6B). However, when HIR1 was also deleted in cac1s strains, but not in a $\operatorname{sir} 1 \Delta$ sas $2 \Delta$ strain, silencing at $H M L$ decreased further (Fig. 6A). These results showed that $\operatorname{cac} 1 \Delta$ and sas $2 \Delta$ were in the same genetic pathway for silencing at $H M L$ in the absence of SIR 1 and that the role of HIR1 was parallel to that of CAC1 but not of SAS2 at $H M L$. The functional epistasis of SAS2 and CAC1 was also found at the telomeres, where loss of telomeric silencing of a sas $2 \Delta$ strain was indistinguishable from a sas $2 \Delta$ cac1s strain (data not shown).

Cac1 has also been shown to play a role at $H M L$ in wild-type strains and at the natural $H M R$ locus (Enomoto and Berman 1998; Kaufman et al. 1998). To test the effect of sas $2 \Delta$ in these contexts, we assayed silencing in a strain with the $A D E 2$ gene inserted at $H M R$. Interestingly sas $2 \Delta$ caused reduced $H M R:: A D E 2$ silencing, as did cac1 1 . The sas $2 \Delta$ cac1 $\Delta$ double-delete strain showed a stronger loss of silencing compared with that of either deletion alone, arguing that the roles of $S A S 2$ and $C A C 1$ were related, but not overlapping, at natural HMR (Fig. 6C).

In MATa strains, cac1 $\Delta$ causes defects in maintenance of $H M L$ silencing, which results in improper cellular arrest in response to $\alpha$-factor (Enomoto and Berman 1998). However, sas $2 \Delta$ did not show schmoo clusters (Fig. 6D), and the deletion of $S A S 2$ in a cac1 1 strain weakened the $\alpha$-factor response of cac1 $\Delta$ cells, arguing that SAS2 played a positive role in $H M L$ silencing that was similar but not functionally overlapping with $C A C 1$.

Interestingly, sas $2 \Delta$ resulted in increased rDNA silencing, whereas cac1s decreases rDNA silencing and enhances recombination. We found that a $\operatorname{sas} 2 \Delta \operatorname{cac} 1 \Delta$ strain showed an intermediate phenotype (Fig. 4B), with both enhanced recombination and a mild increase of rDNA silencing. Taken together, these observations suggested that the recruitment to replicated loci of Sas2 by Cac1 constituted only part of the effect of Sas 2 on silencing and, thus, that SAS-I might also interact with other chromatin assembly proteins at different genetic loci. One such candidate is the nucleosome assembly factor Asf1 (see below).

One interpretation of the interaction of SAS-I and Cac1 is that Sas 2 might directly or indirectly influence the activity of the CAF-I complex. To test this, we investigated the effect of the deletion of SAS2 on the activity of CAF-I. Epitope-tagged Cac2 was immunoprecipitated from wild-type, cac1s, and sas $2 \Delta$ cell extracts and tested for its ability to assemble nucleosomes in a DNA replication-preferential manner during SV40 DNA replication in vitro. In this assay, the formation of nucleosomes is measured by an increase in the negative supercoiling of the purified DNA. Supercoiling activity was found in immunoprecipitates with Cac2 that depended on CAC1 (Fig. 6E) and thus likely represented CAF-I activity. However, no difference in supercoiling activity was observed in $\mathrm{Cac} 2$ immunoprecipitates from sas $2 \Delta$ extracts (Fig. 6E), thus arguing that within the limits of detection in this assay, Sas2 neither inhibited nor enhanced CAF-I activity. 
Figure 6. (A) cac1s and sas $2 \Delta$ were epistatic in $H M L$ silencing in $\operatorname{sir} 1 \Delta$ strains. Stains used in a patch mating assay were MATa $H M L \alpha$ and sir1s cac1s (AEY2204),

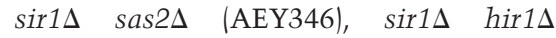
(AEY2236), sir1s sas2 $\Delta$ cac1s (AEY2205),

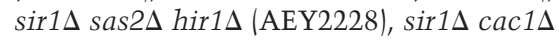

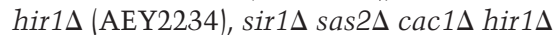
(AEY2230), and sas2s cac1s (AEY2206). MATo his4 as a mating tester. (B) cac2s and $\operatorname{cac} 3 \Delta$ did not cause additional derepression in $\operatorname{sir} 1 \Delta \operatorname{sas} 2 \Delta$ strains. The a mating ability of MATa strains AEY1290

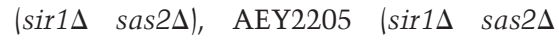

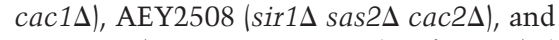
AEY2479 (sir1s sas $2 \Delta$ cac $3 \Delta)$ is shown. $(C)$ sas $2 \Delta$ and cac1s had additive effects on $H M R$ repression. A colony color assay was used to assay repression of the ADE2 gene inserted at $H M R$. Yeast cells were incubated on YM plates supplemented with $10 \%$ of the normal adenine concentration,

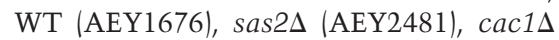
(AEY2483), and sas2s cac1s (AEY2486). (D) The $\alpha$-factor response of MATa $H M L \alpha$ strains was measured by spreading them on complete medium plates containing 40 $\mu \mathrm{g} / \mathrm{mL} \alpha$-factor and incubating them at $23^{\circ} \mathrm{C}$. Approximately 100 cells per strain were scored after $17 \mathrm{~h}$. Schmoo indicates individual cells that formed mating projections and remained arrested; schmoo cluster, individual cells that formed multiple mating projections and eventually divided at least once; and colony, cells that formed colony of round cells and did not appear to respond to $\alpha$-factor. WT (AEY2), sas $2 \Delta$ (AEY266), sir1s (AEY345), cac1s (AEY1403),

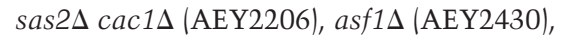

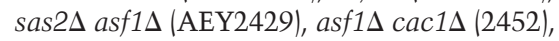
and sas $2 \Delta$ asf1s cac1s (2491). (E) CAF-I activity was not altered in sas $2 \Delta$ strains. (Left) CAF-I activity was immunoprecipitated with myc-Cac2. Immunoprecipitates from AEY1558 or AEY1808 transformed with pAE716 (myc-Cac2) were used in SV40 in vitro replication assays to determine nucleosome assembly activity. The autoradiogram $(t o p)$ of the ethidium bromide stained gel (EtBr, bottom) show immunoprecipitate from a wild-type strain (WT). In the absence of Cac1 (cac1s), immunoprecipitated myc-Cac2 was unable to increase supercoiling. (right) CAF-I activity was unaffected by the absence of Sas2. Increasing amounts of myc-Cac2 immunoprecipitates from wild-type (WT) or sas2 $:: T R P 1$ strains (sas2 $)$ were added to in vitro SV40 replication reactions and assayed as above.

\section{Sas2 interacted with the nucleosome assembly factor Asf1}

The genetic epistasis analysis of Sas 2 with Cac1 (see above) indicated that Sas2, and perhaps the whole SAS-I complex, might interact with other chromatin assembly complexes in yeast. One such candidate is the nucleosome assembly factor Asf1, which has partially overlapping functions with CAF-I (Tyler et al. 1999). Indeed, we observed a two-hybrid interaction between Asf1, Sas2, and Sas5 (Table 1), suggesting that Asf1 interacted with SAS-I. To test the relevance of this interaction in vivo, coimmunoprecipitates were performed on an epitopetagged version of Asf1 with Sas2 and Sas4. Asf1 was coimmunoprecipitated with both Sas2 and Sas4 (Fig. 7A). To further test this connection in vivo, we asked whether the deletion of ASF1 had similar phenotypes as sas $2 \Delta$. Significantly, asf1 $1 \Delta$ caused strong $H M L$ derepression in a $\operatorname{sir} 1 \Delta$ background (Fig. $7 \mathrm{~B}$ ) and suppressed the silencing defect of a mutated $H M R$ allele (Fig. 7C), two 
A

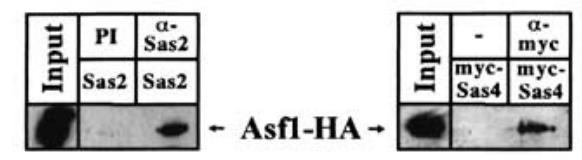

C

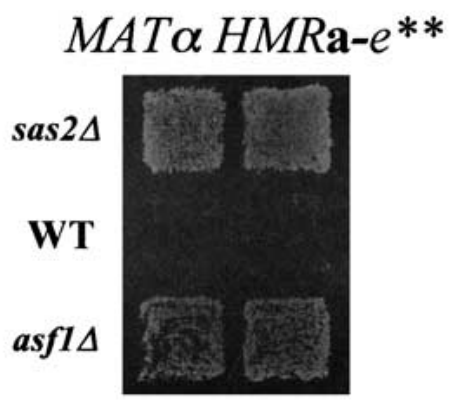

D

\begin{tabular}{|c|c|}
\hline WT & $\operatorname{sir} 1 \Delta \operatorname{sas} 2 \Delta$ \\
\hline $\operatorname{sir} 1 \Delta a s f 1 \Delta$ & $\begin{array}{l}\operatorname{sir} 1 \Delta \operatorname{sas} 2 \Delta \\
\text { asf1 } 1 \Delta\end{array}$ \\
\hline $\begin{array}{l}\operatorname{sir} 1 \Delta \operatorname{cac} 1 \Delta \\
\text { asf1 } 1 \Delta\end{array}$ & $\begin{array}{l}\operatorname{sir} 1 \Delta \operatorname{cac} 1 \Delta \\
\text { asf } 1 \Delta \text { sas } 2 \Delta\end{array}$ \\
\hline
\end{tabular}

B
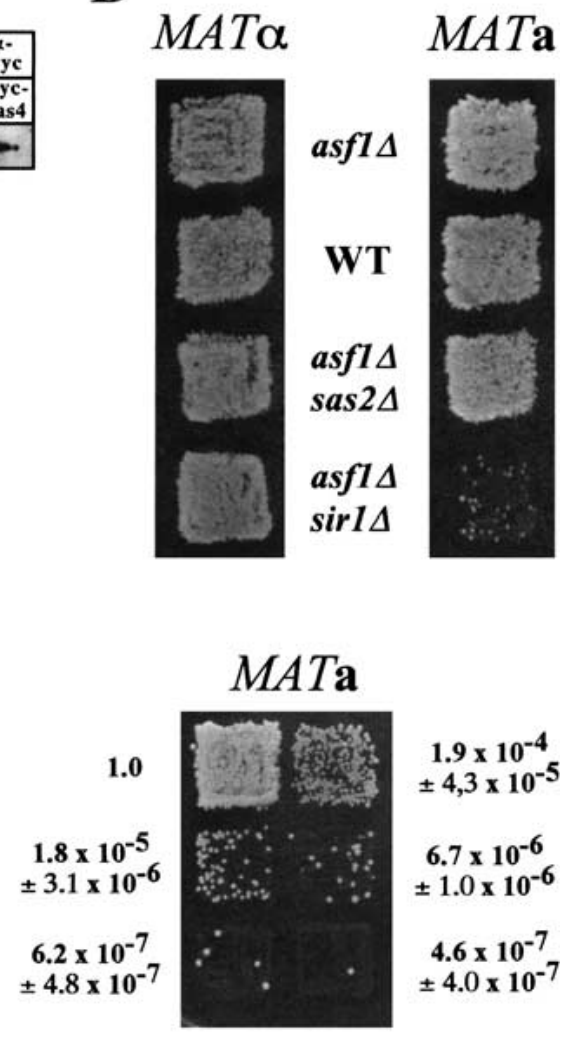

Figure 7. The deletion of ASF1 had the same silencing phenotype as sas $2 \Delta$ at $H M R$ and $H M L$, and Asf1 physically interacted with SAS-I. (A) Asf1 coimmunoprecipitated with Sas2 and Sas4. Extracts from strain AEY2493 (Asf1-HA) expressing Sas2 (pAE240) or myc-Sas4 (pAE612) were immunoprecipitated with $\alpha$-Sas 2 or preimmune serum (PI), or with $\alpha$-myc versus no antibody $(-)$ and immunoblotted with $\alpha$-HA. (B) Disruption of $A S F 1$ caused $H M L$, but not $H M R$,

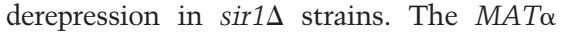
strains AEY1 (WT), AEY2426 (asf1 $\Delta$ ), AEY2490 (sas2 $\Delta$ asf1D), and AEY2428 (asf1s $\operatorname{sir} 1 \Delta)$ were tested for their $\alpha$-mating. Likewise, the a-mating ability of the MATa strains AEY2 (WT), AEY2430 (asf1 $\Delta$ ),

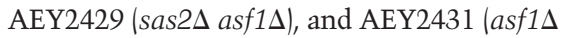
$\operatorname{sir} 1 \Delta)$ is shown. $(C)$ asf $1 \Delta$ restored repression at a mutated $H M R$ allele. Shown is the $\alpha$-mating ability of MAT $\alpha H M R \mathbf{a}-\mathrm{e}^{\star *}$ strains and WT (AEY403), sas2D (AEY474), or asf1s (AEY2363). (D) Effect of combinations of $\operatorname{sir} 1 \Delta$, cac1 $1 \Delta$, asf $1 \Delta$, and sas $2 \Delta$ on $H M L$ silencing. The a-mating ability of the MATa

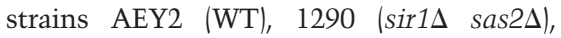

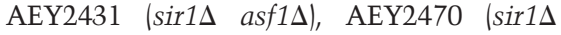
sas2 $2 \Delta$ asf $1 \Delta)$, AEY2471 ( $\operatorname{sir} 1 \Delta$ cac1 $1 \Delta$ asf1 $1 \Delta)$, and AEY2473 (sir1s cac1s asf $1 \Delta$ sas $2 \Delta$ ) was tested. Results of quantitative mating assays are shown relative to a value of 1.0 for AEY2. silencing effects that are also found for sas $2 \Delta$. Furthermore, simultaneous deletion of both ASF1 and SAS2 did not have additive effects on derepression at $H M L$ in $\operatorname{sir} 1 \Delta$ strains (Fig. 7D) and at $H M R$ (Fig. 7B).

If SAS-I performed its role in silencing by being recruited to silenced loci by Asf1 and Cac1, sas $2 \Delta$ should not decrease silencing in a cac1s asf1s strain. Consistent with this, sas $2 \Delta$ did not increase derepression in a $\operatorname{sir} 1 \Delta$ cac1s asf1s strain (Fig. 7D) and in a cac1s asf1s strain in schmoo cluster experiments (Fig. 6D). Taken together, these results argued that the function of Sas2 in $H M L$ silencing was overlapping with both Cacl and Asf1.

\section{Discussion}

The reestablishment of epigenetic imprints in chromatin after DNA replication involves, among others, the restoration of histone acetylation patterns, which play a central role in determining the expression state of genes. The CAF-I complex assembles nucleosomes on DNA in a replication-coupled manner (Kaufman et al. 1997) and has a role in the formation of heterochromatin-like structures in yeast (Enomoto et al. 1997). Here, we found that the large subunit of yeast CAF-I, Cac1, interacted with a protein complex called SAS-I that contained the acetyltransferase homolog Sas2, the cullin family protein Sas4, and the $\mathrm{TAF}_{\mathrm{II}} 30$ homolog Sas5. Whether the interaction of the SAS-I complex with Cacl is direct or alternatively bridged by other proteins can not be concluded at present. The observation that SAS-I interacts with Cacl suggested a functional link between the processes of nucleosome assembly and chromatin acetylation. CAF-I also performs DNA repair coupled chromatin assembly, which is reflected in the sensitivity of yeast cacs mutants to ultraviolet irradiation (Kaufman et al. 1997). However, SAS-I is unlikely to be involved in this aspect of CAF-I function, because $\operatorname{sas} 2 \Delta$, sas $4 \Delta$, and sas $5 \Delta$ strains were not ultraviolet sensitive.

We found that SAS-I only interacted with Cac1, not with other CAF-I subunits, suggesting functions for Cacl separate from CAF-I. A role for a CAF-I subunit independent of the CAF-I complex is also known for Cac3 (Johnston et al. 2001). CAF-I is recruited to the replication fork via the association between Cac1 and PCNA (Shibahara and Stillman 1999). Interestingly, a significantly larger fraction of Cac1 than of Cac2 is associated with chromatin, and chromatin binding of Cac1 but not Cac2 is abrogated by specific mutations in PCNA (Zhang et al. 2000). In light of our results, one interpretation is that $\mathrm{Cacl}$ remains associated with chromatin via PCNA after nucleosome assembly and performs other functions, for example, the recruitment of acetyltransferase complexes and potentially of other chromatin or DNA-modifying activities. In human cells, CAF-I remains associated with chromatin for a longer period of time than necessary for nucleosome assembly, suggesting that CAF-I in these cells may also be involved 
in postreplicative aspects of chromatin formation (Taddei et al. 1999). Interestingly, the large CAF-I subunit has also been invoked in heterochromatin assembly in large eukaryotes, because murine CAF-I interacts with heterochromatin protein 1 (Murzina et al. 1999).

How does CAF-I contribute to gene silencing in yeast? One explanation is that CAF-I activity is required for the formation of ordered nucleosomal structures, which are necessary for efficient silencing. Our results present a parallel model, whereby the SAS-I complex is no longer recruited to freshly replicated chromatin in the absence of Cac1, thus perturbing the reestablishment of epigenetic marks after replication.

The deletion of CAC1 displays milder and, in part, different silencing phenotypes than the deletion of SAS-I components does, arguing that SAS-I also interacts with other chromatin assembly complexes, and we present evidence for an interaction with Asf1. cac1s and asf1s show additive effects in telomeric derepression that are mediated by PCNA (Sharp et al. 2001). Interestingly, a mutation in PCNA (pol30-52) that probably affects all aspects of PCNA function was previously shown to improve silencing at a defective $H M R$ locus, as does sas $2 \Delta$ (Ehrenhofer-Murray et al. 1999), and cause telomeric derepression (S.H. Meijsing, unpubl.). Mutations in PCNA combine the effects of both CAF-I and Asf1, and hence, one explanation for this phenotype is that in the pol3052 mutant, neither assembly factor, and thus no SAS-I, is recruited to the replication fork. Thus, improved silencing at a mutant HMR locus by pol30-52 in essence may be caused by the lack of recruitment of the acetyltransferase complex SAS-I. Furthermore, because asf1D (Singer et al. 1998), but not cac1s (Smith et al. 1999), improves rDNA silencing, as does sas $2 \Delta$, we propose that SAS-I is recruited to rDNA by its association with Asf1. Our observation that rDNA association of Sas2 is reduced in a cac $1 \Delta$ asf $1 \Delta$ strain suggests that chromatin assembly factors may indeed play a role in recruitment of the SAS-I complex to the rDNA.

Interestingly, we found different effects for Asf1 and Hirl on $H M$ silencing. asf1 1 , but not hir1s, caused $H M L$ derepression in $\operatorname{sir} 1 \Delta$ strains (Fig. 6A). In addition, asf1 $\Delta$ suppressed $H M R$ silencing defects, whereas hir1s increased derepression at the same $H M R$ allele (A.E. Ehrenhofer-Murray, unpubl.). Thus, although Asf1 and Hir1 cooperate in some aspects of silencing, they have separate functions, at least in some contexts. Additionally, we found that an $\operatorname{sir} 1 \Delta \operatorname{cac} 1 \Delta$ asf1s strain was more mating defective than a sir1s sas $2 \Delta$ strain, suggesting that Asf1 and Cac1 play roles in silencing independent of the SAS-I complex. This may include recruitment of other silencing factors, a role of CAF-I and Asf1 in the formation of ordered nucleosomal structures, or regulation of histone levels by Asf1 (Sutton et al. 2001), which may all contribute to heterochromatin destabilization.

The acetyltransferase homolog Sas 2 was part of the SAS-I complex that included Sas4 and Sas5, which is supported by the observation that the deletion of any one of the respective genes results in a specific set of silencing phenotypes. Notably, Sas5 is homologous to $\mathrm{TAF}_{\mathrm{II}} 30$, which is a component of the nucleosome acetylation complex NuA3 (John et al. 2000). The catalytic subunit of NuA3 is the MYST family protein Sas3. Thus, there are parallels in the subunit composition between SAS-I and NuA3. Interestingly, both the acetyltransferases Sas2 and Sas3, as well as Sas5 and $\mathrm{TAF}_{\mathrm{II}} 30$, are homologous to human proteins implicated in acute myeloid leukemia, MOZ and MLL/AF-9, suggesting that inappropriate chromatin acetylation plays a crucial role in malignant transformation.

What is the cellular role of Sas2? The resemblance of Sas2 to known acetyltransferases makes it likely that Sas 2 is an acetyltransferase, which is supported by our observation that a mutation of Lys 16 in histone $\mathrm{H} 4$ that imitates the deacetylated state (K16R) showed the same specific set of silencing phenotypes as sas $2 \Delta$. The lack of Sas 2 acetylation activity in vitro (A.E. Ehrenhofer-Murray, unpubl.) suggests that Sas2 function may require additional cofactors or that Sas 2 has an unusual substrate specificity.

It is commonly thought that histone acetylation is correlated with gene activation; and histone deacetylation, with gene repression and transcriptional silencing. However, our data suggest a more complicated model for the interrelationship of acetylation states and gene expression. The deletion of $S A S 2$ results in opposite effects on silencing at the different silenced loci in yeast. Mutated $H M R$ and rDNA repression increased in sas $2 \Delta$, which is in agreement with the notion that repression is improved in the absence of an acetyltransferase. In contrast, sas $2 \Delta$ caused a loss of telomeric silencing, reduced $H M R:: A D E 2$ silencing, and a loss of $H M L$ silencing in $\operatorname{sir} 1 \Delta$ strains. Therefore, by inference, Sas2, and thus acetylation at these loci, was required for silencing. Also, the observation that $\mathrm{H} 4 \mathrm{~K} 16 \mathrm{R}$ causes $H M L$ derepression in $\operatorname{sir} 1 \Delta$ strains and telomeric derepression, despite the fact that it imitates the deacetylated state of $\mathrm{H} 4$, argues that the simplest model for acetylation in silencing does not apply. Significantly, a neutral amino acid substitution at $\mathrm{H} 4 \mathrm{~K} 16$ (K16Q) causes HML derepression (Johnson et al. 1992), whereas an artificial positive charge (K16R) caused derepression only in sir1s strains. One way of explaining these observations is that Lys 16 is temporarily acetylated at the silenced loci and that, under some circumstances, the flexibility and dynamics of this acetylation/deacetylation are required for repression. Thus, loss of this flexibility, either by a mutation in the critical $\mathrm{H} 4$ residue or by the lack of the respective acetyltransferase, results in a loss of silencing. This model also implies that there are subtle differences in the mechanisms of silencing at the silenced loci in yeast, because both sas2 2 and H4 K16R suppressed silencing defects at $H M R$.

In conclusion, we propose a novel model for the reestablishment of histone acetylation patterns after DNA replication. We present data arguing that the recruitment of an acetyltransferase complex, SAS-I, is mediated by its association with Cacl and Asf1. In larger eukaryotes, the histones incorporated into new chromatin carry acetyl groups at lysines 5 and 12 of $\mathrm{H} 4$, an acetylation 
pattern determined by cytoplasmic acetyltransferases. These acetyl groups are subsequently removed from heterochromatin in a trichostatin-sensitive fashion shortly after replication (Taddei et al. 1999), suggesting the involvement of deacetylases in this process. In light of our findings, we propose that CAF-I and Asf1 serve as platforms for chromatin-modifying activities that reestablish epigenetic imprints on replicated chromatin. A future challenge will be to understand how these activities are spatially directed within the genome to ensure the reestablishment of these imprints at the appropriate time and location.

\section{Materials and methods}

\section{Yeast strains and plasmids}

Yeast strains and plasmids used in this study are given in Tables 2 and 3, respectively. Growth and manipulation of yeast was performed according to standard procedures. Gene knockouts with kanMX were performed as described (Wach et al. 1994). Disruption of HIR1 was performed using the PCR-mediated knockout technique, thereby replacing the complete open reading frame by HIS3 sequence, and integrants were selected for using standard genetic techniques. Correct integration in all cases was verified by PCR analysis. The deletion of $S A S 2$ by TRP1 was as described (Ehrenhofer-Murray et al. 1997). The construction of $\mathrm{H} 4$ mutant strains carrying telomeric URA3 was achieved by integrating TEL VII-L ::URA3 (SalI/EcoRI linearized pVII-L URA3-TEL; Gottschling et al. 1990) into strains carrying either HHF1 or HHF1 (K16R) on TRP1 plasmids as their sole source of histone H4. Epitope-tagged versions of Asf1, Cac3, and Sas5 in AEY1558 were constructed as described (Zachariae et al. 1998).

\section{Two-hybrid screening}

A LexA-Sas2 hybrid containing full-length Sas2 was used to screen a yeast two-hybrid library (Durfee et al. 1993). Plasmids from clones that tested positive for expression of both HIS3 and $\beta$-galactosidase were amplified in Escherichia coli, retransformed into the two-hybrid tester strain, and tested for autoactivation and interaction with LexA. Only candidates that specifically interacted with Sas2 were further analyzed.

\section{Antibodies and immunoblotting}

SDS-PAGE and Western blotting procedures were performed according to standard protocols. Anti-epitope antibodies were purchased from Invitrogen $(\alpha-\mathrm{myc})$ and Covance $(\alpha-\mathrm{HA})$. A polyclonal antibody against amino acids $4-148$ of Sas 2 was raised in guinea pigs using standard procedures, and the antibody was used in a 1:40,000 dilution for Western blotting.

\section{Immunoprecipitations}

Yeast extracts from protease deficient strains AEY1558 and AEY1559 were prepared as described by Moazed and Johnson (1996), except that the concentration of $\mathrm{NaCl}$ in the extraction buffer was adjusted to $200 \mathrm{mM}$ and the centrifugated extracts were used directly for subsequent immunoprecipitations. Immunoprecipitations were performed as follows: Antibody was added to the lysate and incubated for $1 \mathrm{~h}$ at $4^{\circ} \mathrm{C}$ with shaking. Subsequently, G-Sepharose 4-FF beads (Pharmacia) were added to the lysate-antibody mix and incubated overnight. Immunoprecipitates were collected by brief centrifugation and washed four times with buffer $\mathrm{L}(125 \mathrm{mM}$ Tris- $\mathrm{HCl}$ at $\mathrm{pH} 7.5,1 \mathrm{mM}$ EDTA, $10 \mathrm{mM} \mathrm{MgOAc}, 100 \mathrm{mM} \mathrm{NaCl}$, and $1 \mathrm{mM}$ DTT). The resulting precipitate was resuspended in SDS sample buffer and analyzed by immunoblotting. When applicable, ethidium bromide was added to the immunoprecipitates at $100 \mu \mathrm{g} / \mathrm{mL}$. CHIPs were performed as described (Hecht and Grunstein 1999). PCR reactions of immunoprecipitated DNA were performed with $1 / 30$ of the precipitated material and 2.5 -fold dilutions thereof. PCR cycles in all cases were 30 cycles of $1 \mathrm{~min}$ at $94^{\circ} \mathrm{C}$ (denaturation), $1 \mathrm{~min}$ at $52^{\circ} \mathrm{C}$ (annealing), and $1 \mathrm{~min}$ at $72^{\circ} \mathrm{C}$ (extension). PCR products were analyzed on $1 \%$ agarose gels. Inverse images of ethidium bromide-stained gels are presented. The primers for the NTS of RDN1 were identical to those in Gotta et al. (1997); those for ACT1 are available on request. For $A C T 1$, no sequences were amplified in the precipitates, but amplifications of the input were weaker than for NTS. Therefore, nested PCR was performed with the primary amplificate (20 cycles).

\section{Gel filtration}

Whole cell lysates for gel-filtration experiments were prepared as described for the immunoprecipitation experiments. A 120 $\mathrm{mL}$ gel filtration column (HiPrep 16/60 Sephacryl S-300 HR; Pharmacia) was equilibrated in buffer A $(125 \mathrm{mM}$ Tris- $\mathrm{HCl}$ at pH 7.5, 1 mM EDTA, 10 mM MgAc, 200 mM NaCl 0.1\% NP-40, $1 \mathrm{mM}$ DTT, and protease inhibitors). The elution profile of the proteins of interest was compared with the elution of marker proteins from a commercially available marker mix (Pharmacia). Proteins from every other fraction were trichloroacetic acid precipitated and tested by immunoblotting.

\section{SV40 DNA replication coupled chromatin assembly assay}

SV40 based DNA replication reactions with HeLa extract, SV40 large $\mathrm{T}$ antigen, pUC-HSO plasmid DNA, and $\left[{ }^{32} \mathrm{P}\right] \mathrm{dCTP}$ were purchased from Chimerx and performed according to the manual instructions, essentially as described (Stillman 1986). CAF-I-dependent replication coupled assembly of nucleosomes was tested in replication reactions by adding immunoprecipitated myc-Cac2. Products were analyzed by gel electrophoresis in $1 \%$ TBE-agarose gels. The incorporation of negative supercoils into the newly replicated DNA was detected by autoradiography of vacuum-dried gels.

\section{Silencing assays}

Expression of the RDN::Ty $1::$ mURA3 and RDN::Ty1::MET15 rDNA reporter constructs were assayed as described (Smith et al. 1999). Silencing of the TEL VII-L::URA3 reporter gene was tested by spotting serial dilutions on 5-FOA plates. Qualitative and quantitative mating assays were performed as described (Ehrenhofer-Murray et al. 1997) using AEY264 (MATa his4) or AEY265 (MAT $\alpha$ his4) as mating tester strains.

\section{Immunofluorescence on yeast cells}

Cells carrying a GFP-Sas2 fusion protein under the control of the GPD promoter on a $2 \mu$-based plasmid (pAE94) were grown to logarithmic phase in liquid selective medium, stained with DAPI $(10 \mu \mathrm{g} / \mathrm{mL})$, and directly visualized under a fluorescence microscope using the FITC filter for GFP. 
Table 2. Yeast strains used in this study

\begin{tabular}{|c|c|c|}
\hline Strain & Genotype & Source $^{\mathrm{a}}$ \\
\hline AEY1 & $\begin{array}{l}\text { MAT } \alpha \text { ade2-1 ura3-1 his3-11,15 } \\
\text { leu2-3, } 112 \text { trp1-1 can1-100 } \\
\text { (=W303-1B) }\end{array}$ & \\
\hline AEY2 & $\begin{array}{l}\text { MATa ade2-1 ura3-1 his3-11,15 } \\
\text { leu2-3, } 112 \text { trp1-1 can1-100 } \\
\text { (=W303-1A) }\end{array}$ & \\
\hline AEY266 & MATa sas $2 \Delta:: T R P 1$ & \\
\hline AEY269 & $M A T \alpha$ sas $2 \Delta:: T R P 1$ & \\
\hline AEY345 & MATa $\operatorname{sir} 1 \Delta:: L E U 2$ & \\
\hline AEY346 & MATa $\operatorname{sir} 1 \Delta:: L E U 2$ sas $2 \Delta:: T R P 1$ & \\
\hline AEY403 & $M A T \alpha H M R \mathbf{a}-\mathrm{e}^{\star \star}$ & \\
\hline AEY474 & $M A T \alpha H M R \mathbf{a}-\mathrm{e}^{\star \star}$ sas $2 \Delta:: T R P 1$ & \\
\hline AEY1017 & MAT $\alpha$ TEL-VIIL::URA3 & J. Berman \\
\hline AEY1190 & $\begin{array}{l}\text { MAT } \alpha \text { TEL-VIIL::URA3 } \\
\text { sas } 2 \Delta:: T R P 1\end{array}$ & \\
\hline AEY1290 & MATa $\operatorname{sir} \Delta:: H I S 3$ sas $2 \Delta:: T R P 1$ & \\
\hline AEY1403 & MATa $\operatorname{cac} 1 \Delta:: L E U 2$ & P. Kamakaka \\
\hline AEY1676 & $M A T \alpha H M R:: A D E 2$ & D. Shore \\
\hline AEY1956 & 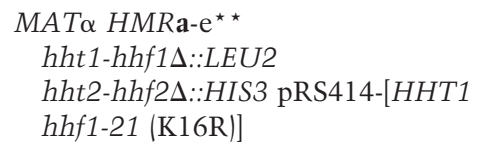 & \\
\hline AEY1974 & 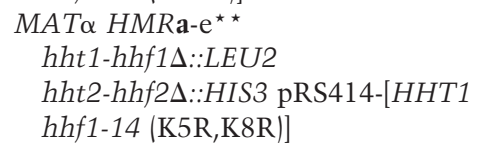 & \\
\hline AEY1976 & 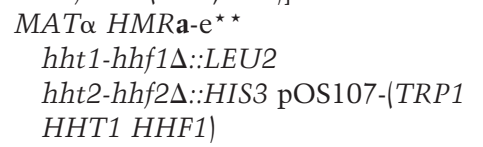 & \\
\hline AEY2197 & 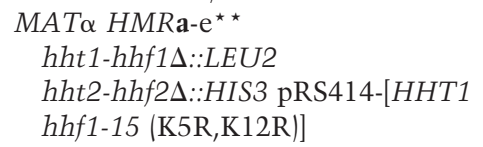 & \\
\hline AEY2204 & 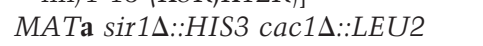 & \\
\hline AEY2205 & $\begin{array}{l}\text { MATa } \operatorname{sir} 1 \Delta:: H I S 3 \text { cac1s::LEU2 } \\
\quad \text { sas } 2 \Delta:: T R P 1\end{array}$ & \\
\hline AEY2206 & MATa $\operatorname{cac} 1 \Delta:: L E U 2$ sas $2 \Delta:: T R P 1$ & \\
\hline AEY2221 & 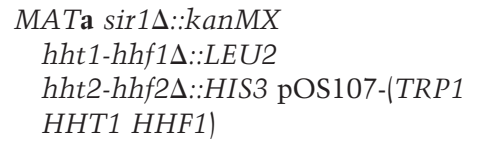 & \\
\hline AEY2222 & 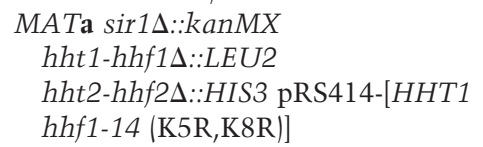 & \\
\hline AEY2223 & 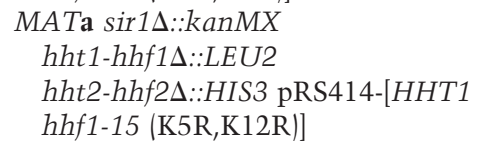 & \\
\hline AEY2224 & 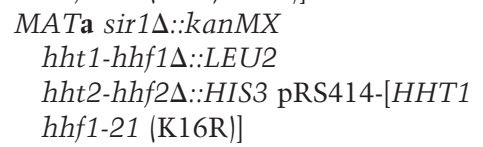 & \\
\hline AEY2228 & 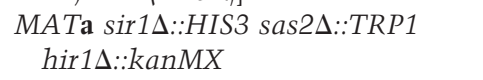 & \\
\hline AEY2230 & 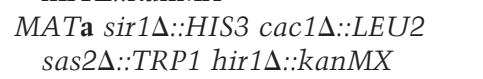 & \\
\hline AEY2234 & 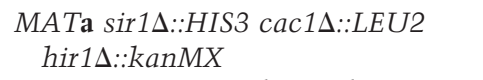 & \\
\hline AEY2236 & 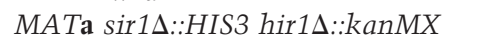 & \\
\hline
\end{tabular}

Table 2. (Continued)

\begin{tabular}{|c|c|c|}
\hline Strain & Genotype & Source ${ }^{\mathrm{a}}$ \\
\hline AEY2302 & $\begin{array}{l}\text { MATa } \\
\quad h h t 1-h h f 1 \Delta:: L E U 2 \text { hht2-hhf2A::HIS3 } \\
\text { TEL-VIIL::URA3 pOS107-(TRP1 } \\
\text { HHT1 HHF1) }\end{array}$ & \\
\hline AEY2304 & 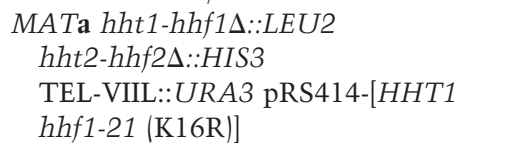 & \\
\hline AEY2363 & $M A T \alpha H M R \mathbf{a}-\mathrm{e}^{\star \star}$ asf $1 \Delta:: \operatorname{kanMX}$ & \\
\hline AEY2426 & $M A T \alpha$ asf $1 \Delta:: \operatorname{kanMX}$ & \\
\hline AEY2428 & $M A T \alpha \operatorname{sir} 1 \Delta::$ LEU2 asf1 $1 \Delta:$ kanMX & \\
\hline AEY2429 & MATa asf1 $::$ kanMX sas $2 \Delta:: T R P 1$ & \\
\hline AEY2430 & MATa asf1 $::$ kanMX & \\
\hline AEY2431 & MATa $\operatorname{sir} 1 \Delta::$ LEU2 asf1 $1:: k a n M X$ & \\
\hline AEY2451 & 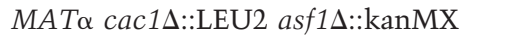 & \\
\hline AEY2452 & MATa $\operatorname{cac} 1 \Delta::$ LEU2 asf1 $::$ kanMX & \\
\hline AEY2470 & 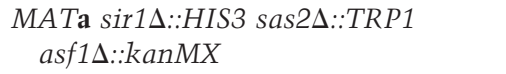 & \\
\hline AEY2471 & 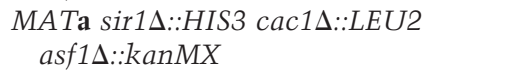 & \\
\hline AEY2473 & $\begin{array}{l}\text { MATa sir1 } 1:: H I S 3 \text { cac1DLEU2 } \\
\text { asf }:: \text { kanMX sas } 2 \Delta:: T R P 1\end{array}$ & \\
\hline AEY2475 & AEY1956 sas2d::kanMX & \\
\hline $\mathrm{AEY} 2479$ & AEY1290 cac3s::kanMX & \\
\hline AEY2481 & AEY1676 sas2 $2:: T R P 1$ & \\
\hline AEY2483 & AEY1676 cac1 $1::$ LEU2 & \\
\hline AEY2485 & $\begin{array}{l}\text { AEY } 1676 \text { sas } 2 \Delta:: T R P 1 \\
\text { cac1 } 1 \Delta:: L E U 2\end{array}$ & \\
\hline AEY2490 & 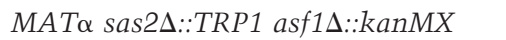 & \\
\hline AEY2491 & $\begin{array}{l}\text { MATa cac1 } 1:: L E U 2 \text { asf } 1 \Delta:: \operatorname{kan} M X \\
\quad \text { sas } 2 \Delta:: T R P 1\end{array}$ & \\
\hline AEY2492 & 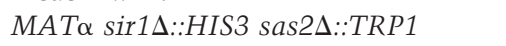 & \\
\hline AEY2508 & AEY1290 cac2s ::kanMX & \\
\hline AEY1558 & $\begin{array}{l}\text { MATa leu2 trp1 ura3-52 prc1-407 } \\
\text { pep4-3 prb1-112 }\end{array}$ & E.W. Jones \\
\hline AEY1559 & AEY1558 sas2A::TRP1 & \\
\hline AEY1808 & AEY1558 cac1s::kanMX & \\
\hline AEY2424 & AEY1559 sas4d::kanMX & \\
\hline AEY2465 & $\begin{array}{l}\text { AEY1558 sas } 4 \Delta:: \text { kanMX } \\
\text { SAS5-3HA::TRP1 }\end{array}$ & \\
\hline AEY2463 & AEY1558 CAC3-9myc::TRP1 & \\
\hline AEY2493 & AEY1558 ASF1-4HA::TRP1 & \\
\hline AEY160 & 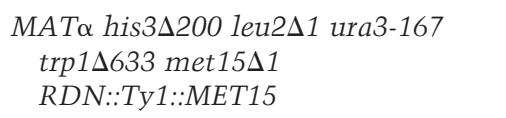 & J. Boeke \\
\hline AEY1195 & AEY160 sas $2 \Delta:: T R P 1$ & \\
\hline AEY1202 & AEY160 sir2A::HIS3 & J. Boeke \\
\hline AEY1755 & AEY160 cac1D::kanMX & \\
\hline AEY1978 & 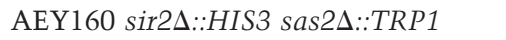 & \\
\hline AEY2487 & AEY1755 sas $2 \Delta:: T R P 1$ & \\
\hline AEY1778 & 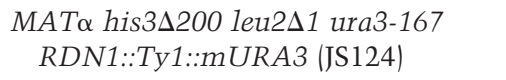 & J. Boeke \\
\hline AEY2279 & AEY1778 sas $2 \Delta:: H I S 3$ & \\
\hline AEY2360 & AEY1778 sas $4 \Delta:: \operatorname{kan} M X$ & \\
\hline AEY2361 & $\mathrm{AEY} 1778$ sas $5 \Delta:: \operatorname{kan} M X$ & \\
\hline
\end{tabular}

${ }^{a}$ Unless indicated otherwise, strains were constructed during the course of this study or were from the laboratory strain collection. Groups of strains between horizontal lines are isogenic. 
Table 3. Plasmids used in this study ${ }^{a}$

\begin{tabular}{|c|c|}
\hline pAE94 & YEp352-GPD ${ }_{\mathrm{p}}$-GFP-SAS2-PGK \\
\hline pAE227 & pRS303-SAS2 \\
\hline pAE230 & pRS303-SAS2-(HAT $\left.{ }^{-}\right)$ \\
\hline pAE240 & YEp351-GPD $-S A S 2-$ PGK $_{\mathrm{t}}$ \\
\hline pAE321 & YEp351-GPD ${ }_{\mathrm{p}}-S A S 2-\left(\mathrm{HAT}^{-}\right)-\mathrm{PGK}_{\mathrm{t}}$ \\
\hline pAE389 & pRS303-SAS2-(Zn-) \\
\hline pAE436 & pBTM117c-LexA-SAS2 \\
\hline pAE439 & pACT2-SAS2-Gal4AD \\
\hline pAE451 & pBTM117c-LexA-SAS4 \\
\hline pAE491 & YEp351-GPD ${ }_{\mathrm{p}}-S A S 2-\left(\mathrm{Zn}^{-}\right)-\mathrm{PGK}_{\mathrm{t}}$ \\
\hline pAE535 & pACT2-ASF1-Gal4AD \\
\hline pAE544 & pRS426-HA-CAC1 \\
\hline pAE612 & pRS426-6myc-SAS4 \\
\hline pAE613 & pRS424-6myc-SAS4 \\
\hline pAE614 & pRS426-6myc-CAC1 \\
\hline pAE625 & pRS426-HA-SAS5 \\
\hline pAE690 & pBTM117c-LexA-SAS5 \\
\hline pAE716 & pRS405-6myc-CAC2 \\
\hline pAE778 & pRS315-6myc-SAS2 \\
\hline pAE779 & pRS316-6myc-SAS4 \\
\hline pAE817 & pBTM117c-LexA-SAS2-(HAT-) \\
\hline pAE 818 & pBTM117c-LexA-SAS2-(Zn- $\left.{ }^{-}\right)$ \\
\hline pAE820 & pRS424-6myc-CAC2 \\
\hline
\end{tabular}

${ }^{\mathrm{a}}$ Cloning details are available from the author upon request.

\section{Acknowledgments}

We wish to thank J. Berman, J. Boeke, D. Gottschling, R. Kamakaka, J. Rine, D. Rivier, S. Schaper, C. Sengstag, D. Shore, M.M. Smith, and E. Wanker for strains and plasmids. We thank S. Seitz for help with plasmid constructions; S. Schaper for critical reading of the manuscript; A. Barduhn, A. König, and K. Vogel for technical assistance; and our laboratory for many stimulating discussions. This research was supported by the Max-Planck-Society.

The publication costs of this article were defrayed in part by payment of page charges. This article must therefore be hereby marked "advertisement" in accordance with 18 USC section 1734 solely to indicate this fact.

\section{References}

Akhtar, A. and Becker, P.B. 2000. Activation of transcription through histone $\mathrm{H} 4$ acetylation by MOF, an acetyltransferase essential for dosage compensation in Drosophila. Mol. Cell 5: 367-375.

- 2001. The histone H4 acetyltransferase MOF uses a $\mathrm{C} 2 \mathrm{HC}$ zinc finger for substrate recognition. EMBO Rep. 2: $113-118$.

Braunstein, M., Sobel, R.E., Allis, C.D., Turner, B.M., and Broach, J.R. 1996. Efficient transcriptional silencing in Saccharomyces cerevisiae requires a heterochromatin histone acetylation pattern. Mol. Cell. Biol. 16: 4349-4356.

Chien, C.T., Bartel, P.L., Sternglanz, R., and Fields, S. 1991. The two-hybrid system: A method to identify and clone genes for proteins that interact with a protein of interest. Proc. Natl. Acad. Sci. 88: 9578-9582.

Durfee, T., Becherer, K., Chen, P.L., Yeh, S.H., Yang, Y., Kilburn, A.E., Lee, W.H., and Elledge, S.J. 1993. The retinoblastoma protein associates with the protein phosphatase type 1 catalytic subunit. Genes \& Dev. 7: 555-569.

Ehrenhofer-Murray, A.E., Rivier, D., and Rine, J. 1997. The role of Sas2, an acetyltransferase homolog of Saccharomyces cer- evisiae, in silencing and ORC function. Genetics 145: 923934.

Ehrenhofer-Murray, A.E., Kamakaka, R.T., and Rine, J. 1999. A role for the replication proteins PCNA, RF-C, polymerase epsilon and Cdc45 in transcriptional silencing in Saccharomyces cerevisiae. Genetics 153: 1171-1182.

Enomoto, S. and Berman, J. 1998. Chromatin assembly factor I contributes to the maintenance, but not the re-establishment, of silencing at the yeast silent mating loci. Genes \& Dev. 12: 219-232.

Enomoto, S., McCune-Zierath, P.D., Gerami-Nejad, M., Sanders, M.A., and Berman, J. 1997. RLF2, a subunit of yeast chromatin assembly factor-I, is required for telomeric chromatin function in vivo. Genes \& Dev. 11: 358-370.

Gotta, M., Strahl-Bolsinger, S., Renauld, H., Laroche, T., Kennedy, B.K., Grunstein, M., and Gasser, S.M. 1997. Localization of Sir2p: The nucleolus as a compartment for silent information regulators. EMBO J. 16: 3243-3255.

Grunstein, M. 1997. Histone acetylation in chromatin structure and transcription. Nature 389: 349-352.

Gottschling, D.E., Aparicio, O.M., Billington, B.L., and Zakian, V.A. 1990. Position effect at $S$. cerevisiae telomeres: Reversible repression of Pol II transcription. Cell 63: 751-762.

Gruss, C., Wu, J., Koller, T., and Sogo, J.M. 1993. Disruption of the nucleosomes at the replication fork. EMBO J. 12: 45334545.

Hecht, A. and Grunstein, M. 1999. Mapping DNA interaction sites of chromosomal proteins using immunoprecipitation and polymerase chain reaction. Methods Enzymol. 304: 399414.

Hecht, A., Laroche, T., Strahl, B.S., Gasser, S.M., and Grunstein, M. 1995. Histone H3 and H4 N-termini interact with SIR3 and SIR4 proteins: A molecular model for the formation of heterochromatin in yeast. Cell 80: 583-592.

John, S., Howe, L., Tafrov, S.T., Grant, P.A., Sternglanz, R., and Workman, J.L. 2000. The something about silencing protein, Sas3, is the catalytic subunit of NuA3, a yTAF II $^{30-c o n t a i n-~}$ ing HAT complex that interacts with the Spt16 subunit of the yeast CP (Cdc68/Pob3)-FACT complex. Genes \& Dev. 14: 1196-1208.

Johnson, L.M., Fisher, A.G., and Grunstein, M. 1992. Identification of a non-basic domain in the histone $\mathrm{H} 4 \mathrm{~N}$-terminus required for repression of the yeast silent mating loci. $E M B O$ J. 11: 2201-2209.

Johnston, S.D., Enomoto, S., Schneper, L., McClellan, M.C., Twu, F., Montgomery, N.D., Haney, S.A., Broach, J.R., and Berman, J. 2001. CAC3(MSI1) suppression of RAS2(G19V) is independent of chromatin assembly factor I and mediated by NPR1. Mol. Cell. Biol. 21: 1784-1794.

Kaufman, P.D., Kobayashi, R., and Stillman, B. 1997. Ultraviolet radiation sensitivity and reduction of telomeric silencing in Saccharomyces cerevisiae cells lacking chromatin assembly factor-I. Genes \& Dev. 11: 345-357.

Kaufman, P.D., Cohen, J.L., and Osley, M.A. 1998. Hir proteins are required for position-dependent gene silencing in Saccharomyces cerevisiae in the absence of chromatin assembly factor I. Mol. Cell. Biol. 18: 4793-4806.

Moazed, D. 2001. Enzymatic activities of Sir2 and chromatin silencing. Curr. Opin. Cell. Biol. 13: 232-238.

Moazed, D. and Johnson, D. 1996. A deubiquitinating enzyme interacts with SIR4 and regulates silencing in S. cerevisiae. Cell 86: 667-677.

Murzina, N., Verreault, A., Laue, E., and Stillman, B. 1999. Heterochromatin dynamics in mouse cells: Interaction between chromatin assembly factor 1 and HP1 proteins. Mol. Cell 4: 529-540. 
Reifsnyder, C., Lowell, J., Clarke, A., and Pillus, L. 1996. Yeast silencing genes and human genes associated with AML and HIV-1 Tat interactions are homologous with acetyltransferases. Nat. Genet. 14: 42-49.

Sharp, J.A., Fouts, E.T., Krawitz, D.C., and Kaufman, P.D. 2001. Yeast histone deposition protein Asflp requires Hir proteins and PCNA for heterochromatic silencing. Curr Biol. 11: 463-473.

Sherman, J.M. and Pillus, L. 1997. An uncertain silence. Trends Genet. 13: 308-313.

Shibahara, K. and Stillman, B. 1999. Replication-dependent marking of DNA by PCNA facilitates CAF-1-coupled inheritance of chromatin. Cell 96: 575-585.

Singer, M.S., Kahana, A., Wolf, A.J., Meisinger, L.L., Peterson, S.E., Goggin, C., Mahowald, M., and Gottschling, D.E. 1998. Identification of high-copy disruptors of telomeric silencing in Saccharomyces cerevisiae. Genetics 150: 613-632.

Smith, J.S. and Boeke, J.D. 1997. An unusual form of transcriptional silencing in yeast ribosomal DNA. Genes \& Dev. 11: $241-254$.

Smith, J.S., Caputo, E., and Boeke, J.D. 1999. A genetic screen for ribosomal DNA silencing defects identifies multiple DNA replication and chromatin-modulating factors. Mol. Cell. Biol. 19: 3184-3197.

Smith, S. and Stillman, B. 1991. Stepwise assembly of chromatin during DNA replication in vitro. EMBO J. 10: 971-980.

Sterner, D.E. and Berger, S.L. 2000. Acetylation of histones and transcription-related factors. Microbiol. Mol. Biol. Rev. 64: 435-459.

Stillman, B. 1986. Chromatin assembly during SV40 DNA replication in vitro. Cell 45: 555-565.

Sutton, A., Bucaria, J., Osley, M.A., and Sternglanz, R. 2001. Yeast Asfl protein is required for cell cycle regulation of histone gene transcription. Genetics 158: 587-596.

Taddei, A., Roche, D., Sibarita, J.B., Turner, B.M., and Almouzni, G. 1999. Duplication and maintenance of heterochromatin domains. J. Cell. Biol. 147: 1153-1166.

Takechi, S. and Nakayama, T. 1999. Sas3 is a histone acetyltransferase and requires a zinc finger motif. Biochem. Biophys. Res. Commun. 266: 405-410.

Tchenio, T., Casella, J.F., and Heidmann, T. 2001. A truncated form of the human CAF-1 p150 subunit impairs the maintenance of transcriptional gene silencing in mammalian cells. Mol. Cell. Biol. 21: 1953-1961.

Tyler, J.K., Adams, C.R., Chen, S.R., Kobayashi, R., Kamakaka, R.T., and Kadonaga, J.T. 1999. The RCAF complex mediates chromatin assembly during DNA replication and repair. $\mathrm{Na}$ ture 402: 555-560.

Wach, A., Brachat, A., Pohlmann, R., and Philippsen, P. 1994. New heterologous modules for classical or PCR-based gene disruptions in Saccharomyces cerevisiae. Yeast 10: 1793 1808.

Xu, E.Y., Kim, S., and Rivier, D.H. 1999. SAS4 and SAS5 are locus-specific regulators of silencing in Saccharomyces cerevisiae. Genetics 153: 25-33.

Zachariae, W., Shevchenko, A., Andrews, P.D., Ciosk, R., Galova, M., Stark, M.J., Mann, M., and Nasmyth, K. 1998. Mass spectrometric analysis of the anaphase-promoting complex from yeast: identification of a subunit related to cullins. Science 279: 1216-1219.

Zhang, Z., Shibahara, K., and Stillman, B. 2000. PCNA connects DNA replication to epigenetic inheritance in yeast. Nature 408: $221-225$. 


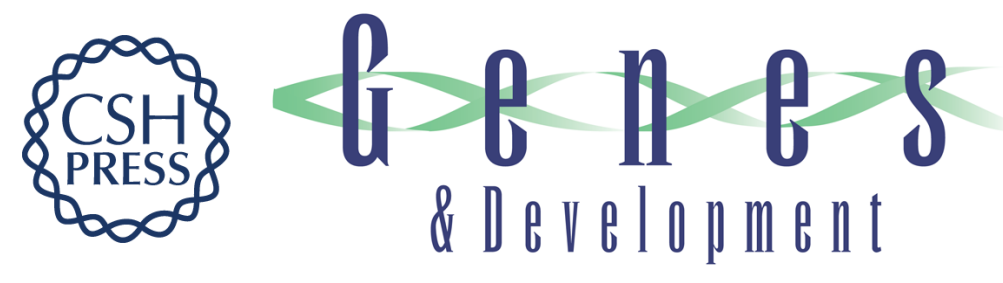

\section{The silencing complex SAS-I links histone acetylation to the assembly of repressed chromatin by CAF-I and Asf1 in Saccharomyces cerevisiae}

Sebastiaan H. Meijsing and Ann E. Ehrenhofer-Murray

Genes Dev. 2001, 15:

Access the most recent version at doi:10.1101/gad.929001

\section{References This article cites 42 articles, 21 of which can be accessed free at: http://genesdev.cshlp.org/content/15/23/3169.full.html\#ref-list-1}

\section{License}

Email Alerting

Service

Receive free email alerts when new articles cite this article - sign up in the box at the top right corner of the article or click here.

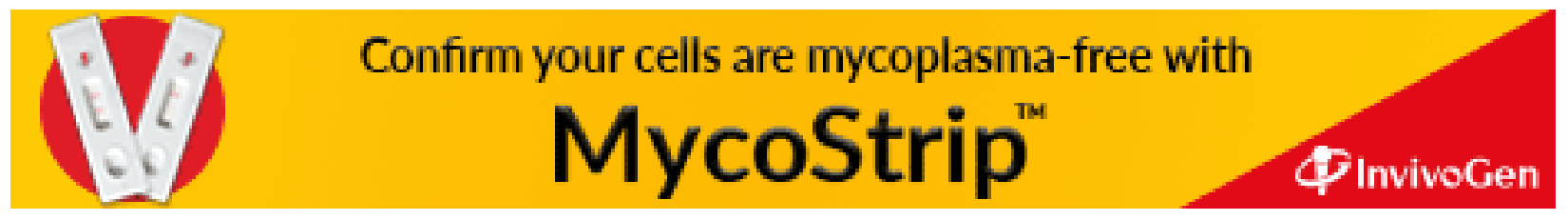

